\title{
ON ACCESSIBLE SUBRINGS OF ASSOCIATIVE RINGS
}

\author{
by R. R. ANDRUSZKIEWICZ
}

(Received 1st March 1990)

\begin{abstract}
We describe for every natural $n$ the class of rings $R$ such that if $R$ is an accessible (left accessible) subring of a ring then $R$ is an $n$-accessible ( $n$-left-accessible) subring of the ring. This is connected with the problem of the termination of Kurosh's construction of the lower (lower strong) radical. The result for $n=2$ was obtained by Sands in a connection with some other questions.
\end{abstract}

1980 Mathematics subject classification (1985 Revision): 16A21.

It was unknown for a long time whether for every natural number $n \geqq 2$ there exists a homomorphically closed class $\mathscr{M}$ of rings such that $\mathscr{M}_{n-2} \neq \mathscr{M}_{n-1}=\mathscr{M}_{n}$, where $\mathscr{M}_{i}$ denotes the $i$ th class in the Kurosh's chain of rings determined by $\mathscr{M}$ (cf. [8]). The problem was solved positively in [1] and then many authors contributed to the topic obtaining several related results (cf. $[3,4,5,9]$ ). It is not difficult to observe (Proposition 3) that the original problem is equivalent to the question whether for every natural number $n \geqq 2$ there exists a homomorphically closed class $\mathscr{M}$ of rings such that

$(\rho)_{n}$ : if a ring $R$ contains a non-zero $n$-accessible subring in $\mathscr{M}$ then $R$ contains a non-zero $n-1$-accessible subring in $\mathscr{M}$.

In this paper we study a related question. Namely we describe for every $n \geqq 2$ the class $\mathbb{l}=\{A \mid$ if $S \cong A$ is an $n$-accessible subring of $R$ then $S$ is an $n-1$-accessible subring of $R\}$, which is naturally connected with a description of rings $A$ such that the class $\{A\}$ satisfies $(\rho)_{n}$. We also describe the class $\mathbb{L}_{n}=\{A \mid$ if $S \cong A$ is an $n$-left-accessible subring of $R$ then $S$ is an $n-1$-left-accessible subring of $R\}$, which is related to the Kurosh's construction of the lower left strong radicals (cf. [5]). Our results generalize Sands' Theorem 1 of [6] which says (in our terminology) that $\mathbb{Z}_{2}=\mathbb{L}_{2}=$ the class of idempotent rings. It is worthy to mention that Sands obtained his result studying some other questions of associative rings.

All rings considered in the paper are associative. Given a ring $R$ we denote:

$R^{+}$- the additive group of $R$.

$R^{0}$ - the trivial ring defined on the group $R^{+}$.

$R^{*}$ - the ring $R$ if $R$ has an identity element and the usual extension of $R$ to a ring with identity by the ring of integers $\mathbb{Z}$ in another case.

We use $I \triangleleft R(I<R)$ to denote that $I$ is an ideal (left ideal) of $R$.

A subring $A$ of a ring $R$ is called an $n$-accessible ( $n$-left-accessible) subring of $R$ if 
there exist subrings $R_{0}, R_{1}, \ldots, R_{n}$ of $R$ such that $R_{n}=A, R_{0}=R$ and $R_{i} \triangleleft R_{i-1}$ $\left(R_{i}<R_{i-1}\right)$ for $i=1,2, \ldots, n$. One can easily check that these subrings are characterised as follows:

Proposition 1. Let $A$ be a subring of a ring $R$. Then

(i) $A$ is an n-left-accessible subring of $R$ if and only if $R A^{n} \subseteq A$;

(ii) $A$ is an $n$-accessible subring of $R$ if and only if $R_{n}=A$, where $R_{0}=R$ and $R_{i+1}=R_{i}^{*} A R_{i}^{*}$ for $i=0,1,2, \ldots$

Let $\mathscr{M}$ be a homomorphically closed class of rings. Then the class $\mathscr{M}_{1}=\{R \mid$ every nonzero homomorphic image of $R$ contains a nonzero ideal $I$ which is in $\mathscr{M}\}$ is homomorphically closed as well. By induction for every natural number $n \geqq 2$, the class

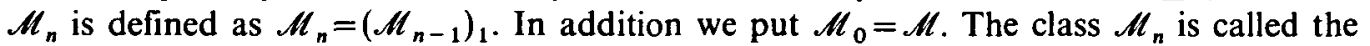
nth Kurosh's class determined by $\mathscr{M}$.

The following properties of classes $\mathscr{M}_{n}$ were established in [2].

Proposition 2. (i) $A$ ring $R$ is in $\mathscr{M}_{n}$ if and only if every nonzero homomorphic image of $R$ contains a nonzero $n$-accessible subring which is in $\mathscr{M}$.

(ii) If $A \in \mathscr{M}$ is a nonzero $n$-accessible subring of a ring $R$ then $R^{*} A R^{*} \in M_{n-1}$.

Corollary. Let $\mathscr{M}$ be a homomorphically closed class of rings. Then for every natural number $n>1$

(i) if $\mathscr{M}$ satisfies $(\rho)_{n}$ then $\mathscr{M}_{n-1}=\mathscr{M}_{n}$;

(ii) if $\mathscr{M}_{n-2}=\mathscr{M}_{n-1}$ then $\mathscr{M}$ satisfies $(\rho)_{n}$;

(iii) $\mathscr{M}$ satisfies $(\rho)_{n+1}$ if and only if $\mathscr{M}_{1}$ satisfies $(\rho)_{n}$.

Proof. (i) is an immediate consequence of Proposition 2(i).

(ii) Suppose that $A \in \mathscr{M}$ is a nonzero $n$-accessible subring of $R$. By Proposition 2(ii), $R^{*} A R^{*} \in \mathscr{M}_{n}$. Since $\mathscr{M}_{n-2}=\mathscr{M}_{n-1}$, we have also $\mathscr{M}_{n-2}=\mathscr{M}_{n}$ so $R^{*} A R^{*} \in \mathscr{M}_{n-2}$. By Proposition 2(i) $R^{*} A R^{*}$ contains a nonzero $n-2$-accessible subring $B \in \mathscr{M}$. However $R^{*} A R^{*}$ is an ideal of $R$, so $B$ is an $n-1$-accessible subring of $R$.

(iii) Let $A \in \mathscr{M}_{1}$ be a nonzero $n$-accessible subring of a ring $R$. Since $A \in \mathscr{M}_{1}, A$ contains a nonzero ideal $I \in \mathscr{M}$. Obviously $I$ is an $n+1$-accessible subring of $R$. Hence, since $\mathscr{M}$ satisfies $(\rho)_{n+1}, R$ contains a nonzero $n$-accessible subring $B \in \mathscr{M}$. Thus there exist subrings $B=B_{n} \triangleleft B_{n-1} \triangleleft B_{n-2} \triangleleft \cdots \triangleleft B_{0}=R$. By Proposition 2(ii), $C=$ $B_{n-2}^{*} B B_{n-2}^{*} \in \mathscr{M}_{1}$. Obviously $C$ is a nonzero $n-1$-accessible subring of $R$.

Suppose now that $\mathscr{M}_{1}$ satisfies $(\rho)_{n}$. Let $A=A_{n+1} \triangleleft \cdots \triangleleft A_{0}=R$ where $0 \neq A \in \mathscr{M}$. Then by Proposition 2(ii) $B=A_{n-1}^{*} A A_{n-1}^{*} \in \mathscr{M}_{1}$ is an $n$-accessible subring of $R$. Hence, since $\mathscr{M}_{1}$ satisfies $(\rho)_{n}, R$ contains a nonzero $n-1$-accessible subring $C \in \mathscr{M}_{1}$. Since $C \in \mathscr{M}_{1}, C$ contains a nonzero ideal $l \in \mathscr{M}$. Obviously $I$ is a nonzero $n$-accessible subring of $R$. 
The following example shows that the converse to Corollary (i) does not hold for $n=2$. We have not been able to construct similar examples for $n>2$.

Example. Let $\mathscr{M}=\{R\} \cup\{0\}$, where $R$ is the ring with trivial multiplication on the quasicyclic group $C\left(p^{\infty}\right)$. It is easy to check that $\mathscr{M}_{1}$ is equal to the class of all rings whose additive groups are divisible $p$-groups. Thus $\mathscr{M}_{1}=\mathscr{M}_{2}$. Observe however that $\mathscr{M}$ does not satisfy $(\rho)_{2}$. Namely

$$
\left(\begin{array}{ll}
R & 0 \\
0 & 0
\end{array}\right) \triangleleft\left(\begin{array}{ll}
R & R \\
R & R
\end{array}\right) \triangleleft\left(\begin{array}{ll}
R^{*} & R^{*} \\
R^{*} & R^{*}
\end{array}\right)=A .
$$

Every ideal of $A$ is of the form $\left(\begin{array}{ll}I & I \\ I & I\end{array}\right)$, where $I$ is an ideal of $R^{*}$. This easily shows that no ideal of $A$ is isomorphic to $R$.

Proposition 3. The following are equivalent:

(i) for every natural number $n$ there exists a homomorphically closed class $\mathscr{M}$ such that $\mathscr{M}_{n-1} \neq \mathscr{M}_{n}=\mathscr{M}_{n+1}$;

(ii) for every natural number $n$ there exists a homomorphically closed class $\mathscr{M}$ satisfying $(\rho)_{n+2}$ but not $(\rho)_{n+1}$.

Proof. $(i) \Rightarrow($ ii). Let $n$ be a natural number and $\mathscr{M}$ a homomorphically closed class of rings such that $\mathscr{M}_{n} \neq \mathscr{M}_{n+1}=\mathscr{M}_{n+2}$. By the Corollary, $\mathscr{M}$ satisfies $(\rho)_{n+3}$ but not $(\rho)_{n+1}$. If $\mathscr{M}$ satisfies $(\rho)_{n+2}$ then (ii) is satisfied. If $\mathscr{M}$ does not satisfy $(\rho)_{n+2}$ then by Corollary (iii), $\mathscr{M}_{1}$ satisfies $(\rho)_{n+2}$ but not $(\rho)_{n+1}$, so again (ii) is satisfied.

(ii) $\Rightarrow(i)$. Let $n$ be a natural number and $\mathscr{M}$ a homomorphically closed class of rings which satisfies $(\rho)_{n+2}$ but not $(\rho)_{n+1}$. By the Corollary, $\mathscr{M}_{n-1} \neq \mathscr{M}_{n+1}=\mathscr{M}_{n+2}$. If $\mathscr{M}_{n}=\mathscr{M}_{n+1}$ then (i) is satisfied. If $\mathscr{M}_{n} \neq \mathscr{M}_{n+1}$ then $\left(\mathscr{M}_{1}\right)_{n-1} \subseteq\left(\mathscr{M}_{1}\right)_{n}=\left(\mathscr{M}_{1}\right)_{n+1}$, so again (i) is satisfied.

Now we pass on to study classes $\mathbb{Q}_{n}$ and $\mathbb{Q}_{n}$.

Theorem 1. For every integer $n \geqq 2, A \in \mathbb{L}_{n}$ if and only if $A^{n}=A^{n-1}$.

Proof. Assuming that $A^{n}=A^{n-1}$ and applying Proposition 1(i) one gets immediately that $A \in \mathbb{R}_{n}$.

Suppose now that $A \in \mathbb{R}_{n}$. The result is clear if $A$ has an identity. Thus suppose that $A$ is a ring without identity. Observe that for a right $A$-module $M$, the set $\left(\begin{array}{ll}A & 0 \\ M & 0\end{array}\right)$ of matrices is a ring under the usual matrix operations and 


$$
\left(\begin{array}{ll}
A & 0 \\
M & 0
\end{array}\right) \cdot\left(\begin{array}{ll}
A & 0 \\
0 & 0
\end{array}\right)^{k}=\left(\begin{array}{ll}
A & 0 \\
M & 0
\end{array}\right) \cdot\left(\begin{array}{ll}
A^{k} & 0 \\
0 & 0
\end{array}\right)=\left(\begin{array}{ll}
A^{k+1} & 0 \\
M A^{k} & 0
\end{array}\right)
$$

Thus by Proposition 1(i), $\left(\begin{array}{ll}A & 0 \\ 0 & 0\end{array}\right)$ is a $k$-left-accessible subring of the ring $\left(\begin{array}{ll}A & 0 \\ M & 0\end{array}\right)$ if and only if $M A^{k}=0$. Put $M=A^{*} / A^{n}$. Clearly $M A^{n}=0$, so $\left(\begin{array}{ll}A & 0 \\ 0 & 0\end{array}\right)$ is an $n$-left-accessible subring of the ring $\left(\begin{array}{ll}A & 0 \\ M & 0\end{array}\right)$. Since $\left(\begin{array}{ll}A & 0 \\ 0 & 0\end{array}\right) \cong A \in \mathbb{L}_{n}$ we have that $\left(\begin{array}{ll}A & 0 \\ 0 & 0\end{array}\right)$ is an $n$-1-left-accessible subring of $\left(\begin{array}{ll}A & 0 \\ M & 0\end{array}\right)$. Consequently $\left(A^{*} / A^{n}\right) A^{n-1}=M A^{n-1}=0$, which implies that $A^{n-1}=A^{n}$. The result follows.

It is clear that every ring $R$ such that $R^{n}=0$ but $R^{n-1} \neq 0$ belongs to $\mathbb{L}_{n+1}-\mathbb{L}_{n}$, so all the classes $\mathbb{L}_{n}$ are distinct.

Remark. Observed that for $M=A^{*} / A^{2}$,

$$
\left(\begin{array}{ll}
A & 0 \\
0 & 0
\end{array}\right) \triangleleft\left(\begin{array}{ll}
A & 0 \\
A / A^{2} & 0
\end{array}\right) \triangleleft\left(\begin{array}{ll}
A & 0 \\
M & 0
\end{array}\right)
$$

Now applying the arguments used above one can easily get Theorem 1 of [6]. However it is not difficult to check that the subrings appearing here are isomorphic (if $1 \notin A$ ) to the ones used in [6], so this is only a more visible form of the same proof.

Now we will describe the classes $0_{n}$. To get this we need several auxiliary lemmas.

Lemma 1 ([7]). For every radical $S$ and each ring $R$ if $R / R^{2} \in S$ then $R^{n} / R^{n+1} \in S$ for every $n=1,2, \ldots$.

Lemma 2. Suppose that $I \triangleleft R$ and $R^{2} \subseteq I$. If $R \in \mathbb{0}_{n}$ then $R / I \in \mathbb{0}_{n}$.

Proof. If $R / I$ is an $n$-accessible subring of a ring $A$ then $R \oplus R / I$ is an $n$-accessible subring of the ring $R \oplus A$. Let us observe that, since $R^{2} \subseteq l, S=\{(r, r+l) \mid r \in R\}$ is an ideal of $R \oplus R / I$. Clearly $S \cong R \in \mathbb{0}_{n} \subseteq \mathbb{0}_{n+1}$. This and the fact that $S$ is an $n+1$-accessible subring of the ring $R \oplus A$, imply that $S$ is an $n$-1-accessible subring of $R \oplus A$. Applying the natural projection of $R \oplus A$ onto $A$ one gets that $R / I$ is an $n-1$-accessible subring of $A$.

The following lemma can be proved by an easy induction. 
Lemma 3. Let $A$ be a subring of a ring $R$ such that $A^{2}=0$ and $R_{0}=R, R_{i+1}=R_{i}^{*} A R_{i}^{*}$, $i=0,1, \ldots$. Then for every $k=0,1, \ldots, A R_{k} A=(A R)^{2^{k}} A$.

Lemma 4. No class $\mathbb{Q}_{n}$ contains $K^{0}$, where $K$ is a field.

Proof. By the Remark the result is clear for $n=2$. Suppose now that $n \geqq 3$ and put $P=K[x] /\left(x^{m+1}\right)$, where $m=2^{n-2}$. Let $R$ be the ring of all $2 \times 2$-matrices over $P$ and

$$
A=\left\{\left(\begin{array}{rr}
k u & k u \\
-k u & -k u
\end{array}\right) \mid k \in K\right\}
$$

where $u=x+\left(x^{m+1}\right)$. Clearly $A \cong K^{0}$. We claim that $A$ is an $n$-accessible but not $n-1$-accessible subring of $R$. For, let $R_{0}=R$ and $R_{i+1}=R_{i}^{*} A R_{i}^{*}, i=0,1, \ldots$. Since

$$
A R \subseteq\left\{\left(\begin{array}{c}
u r_{1} u r_{2} \\
u r_{3} u r_{4}
\end{array}\right) \mid r_{i} \in P\right\}
$$

and $u^{m+1}=0,(A R)^{m} A=0$. Thus by Lemma $3, A R_{n-2} A=0$. This implies that $A R_{n-1}=$ $R_{n-1} A=0$, so $R_{n}=A$. Consequently Proposition 1(ii) implies that $A$ is an $n$-accessible subring of $R$. Suppose now that $A$ is an $n-1$-accessible subring of $R$. Then by Proposition 1(ii), $R_{n-1}=A$ and consequently $A R_{n-2} \subseteq A$. Since $R_{i} \subseteq R_{1}$ for every $i \geqq 1$ and $n-2 \geqq 1$ (as $n \geqq 3$ ), $A R_{n-2} \subseteq A R_{1} \cap A$.

Now

$$
A R_{1} \subseteq\left\{\left(\begin{array}{c}
u^{2} r_{1}, u^{2} r_{2} \\
u^{2} r_{3}, u^{2} r_{4}
\end{array}\right) \mid r_{i} \in P\right\}
$$

so by the definition of $A$ one gets $A R_{1} \cap A=0$. Consequently $A R_{n-2}=0$ and $A R_{n-3} A=$ 0 . Hence by Lemma $3,(A R)^{2^{n-3}} A=0$. Observe that

$$
\alpha=\left(\begin{array}{rr}
u & 0 \\
-u & 0
\end{array}\right)=\left(\begin{array}{rr}
u & u \\
-u & -u
\end{array}\right)\left(\begin{array}{ll}
1 & 0 \\
0 & 0
\end{array}\right) \in A R \quad \text { and } \quad \alpha^{s}=\left(\begin{array}{rr}
u^{s} & 0 \\
-u^{s} & 0
\end{array}\right)
$$

for every $s$. Thus for $s=2^{n-3}+1, \alpha^{s} \neq 0$. On the other hand $\alpha^{s} \in(A R)^{s}=(A R)^{2^{n-3}} A R=0$, a contradiction.

Now we prove the main result of the paper.

Theorem 2. For every natural number $n \geqq 3$ the following are equivalent:

(i) $R \in 0_{n}$,

(ii) the group $\left(R / R^{2}\right)^{+}$is divisible and torsion. 
Proof. Suppose that $R \in \mathbb{I}_{n}$. Applying Lemma 2 one can assume that $R^{2}=0$ and every homomorphic image of $R$ belongs to $I_{n}$. Now if for a prime $p, R / p R \neq 0$ then $R$ can be homomorphically mapped onto $(\mathbb{Z} / p \mathbb{Z})^{0}$. This contradicts Lemma 4 . Hence for every prime $p, p R=R$, so the group $R^{+}$is divisible. Now if the group $R^{+}$were not torsion it could be homomorphically mapped onto $\mathbb{Q}^{+}$, where $\mathbb{Q}$ is the field of rational numbers. This would imply that $\mathbb{Q}^{0} \in I_{n}$, which contradicts Lemma 4.

Suppose now that the group $\left(R / R^{2}\right)^{+}$is divisible and torsion. Applying Lemma 1 and the fact the class of rings with divisible and torsion additive groups is radical one gets that the group $\left(R / R^{3}\right)^{+}$is divisible and torsion. However, the multiplication in a ring with divisible and torsion additive group is trivial, so $\left(R / R^{3}\right)^{2}=0$. Thus $R^{2}=R^{3}$ and the ring $R^{2}$ is idempotent. If $R$ is an accessible subring of a ring $P$ then $R^{2}$ is also such a subring of $P$. Applying Andrunakievich's Lemma and the fact that $R^{2}$ is an idempotent ring one gets that $R^{2}$ is an ideal of $P$. Since the group $\left(R / R^{2}\right)^{+}$is divisible and torsion, the additive group of the ideal $I$ of $P / R^{2}$ generated by $R / R^{2}$ is divisible and torsion as well, so in particular $I^{2}=0$. Consequently $R / R^{2}$ is a 2 -accessible subring of $P / R^{2}$. Thus $R$ is an $n-1$-accessible subring of $P$. The result follows.

As an easy consequence of Theorem 2 one gets that $0_{3}=0_{4}=0_{5}=\cdots=$ the lower


class of rings with divisible and torsion additive groups (both these classes are radical). Recall that by the Remark, $\mathbb{\nabla}=\mathbb{0}_{2}=\mathbb{Q}_{2}$. Observe however that $\mathbb{Q}_{2} \neq \mathbb{0}_{3}$ and $\mathbb{0}_{3} \neq \mathbb{1}_{3}$. It is an easy consequence of the fact that if $R$ is an idempotent ring and $A$ is the ring with trivial multiplication on an additive group $A^{+}$then $R \oplus A \in \mathbb{L}_{3}$ and $R \oplus A \in \mathbb{V}_{3}$ if and only if the group $A^{+}$is divisible and torsion. The following example shows that not every ring of $\mathrm{O}_{3}$ is a direct sum of an idempotent ring and a ring with trivial multiplication.

Example. Let $M$ be a non-zero abelian torsion and divisible group. Observe that the set

$$
R=\left(\begin{array}{lll}
0 & M & M \\
0 & 0 & \mathbb{Z} \\
0 & 0 & \mathbb{Z}
\end{array}\right)
$$

of matrices with the natural operations is a ring such that

$$
R^{2}=\left(\begin{array}{lll}
0 & 0 & M \\
0 & 0 & \mathbb{Z} \\
0 & 0 & \mathbb{Z}
\end{array}\right)
$$

and $\left(R / R^{2}\right)^{+} \cong M$. Thus $R \in \mathbb{0}_{3}$. Moreover, if $I$ is an ideal of $R$ and 


$$
\left(\begin{array}{lll}
0 & m & m_{1} \\
0 & 0 & z_{1} \\
0 & 0 & z_{2}
\end{array}\right) \in I
$$

then

$$
=\left(\begin{array}{lll}
0 & 0 & m \\
0 & 0 & 0 \\
0 & 0 & 0
\end{array}\right)=\left(\begin{array}{lll}
0 & m & m_{1} \\
0 & 0 & z_{1} \\
0 & 0 & z_{2}
\end{array}\right)\left(\begin{array}{lll}
0 & 0 & 0 \\
0 & 0 & 1 \\
0 & 0 & 0
\end{array}\right) \in I,
$$

so if $m \neq 0$ then $I \cap R^{2} \neq 0$. This shows that $R$ contains no ideal $I$ such that $R^{2} \oplus I=R$. Hence in particular $R$ is not a direct sum of an idempotent ring and a ring with trivial multiplication.

Acknowledgement. I am grateful to Professor E. R. Puczyłowski for his valuable suggestions during the preparation of the paper.

\section{REFERENCES}

1. K. I. BEIDAR, A chain of Kurosh may have an arbitrary finite length, Czechoslovak Math. J. 32, (1982), 418-422.

2. A. Heinicke, A note on lower radical constructions for associative rings, Canad. Math. Bull. 11 (1968), 23-30.

3. Liv Shao Xue, Luo Yun lun, Tang Ai Ping, Xiao Jie and Guo Jin Yun, Some results on modules and rings, Bull. Soc. Math. Belg. Sér. B 39 (1987), 181-193.

4. I. V. L'vov, A. V. Sidorow, Stabilization of Kurosh chains, Mat. Zametki 36 (1984), 815-821 (in Russian).

5. E. R. Puczylowskı, On a question concerning strong radicals of associative rings, Quaestiones Math. 10 (1987), 321-338.

6. A. D. SANDS, On ideals in over-rings, Publ. Math. Debrecen 35 (1988), 273-279.

7. A. D. Sands, Radicals and one-sided ideals, Proc. Roy. Soc. Edinburgh Sect. A 103 (1986), 241-251.

8. A. SUlinski, T. ANDerson and N. Divinsky, Lower radical properties of associative and alternative rings, J. London Math. Soc. 41 (1966), 417-424.

9. J. F. WATtERS, On the lower radical construction for algebras, preprint.

InSTITUTE OF Mathematics

UNIVERSITY OF WARSAW

Bialystok Division

AKademicka 2

15-267 BialystoK

Poland 